\title{
Optical Photometry of LMXBs: UW CrB (=MS 1603+260) and V1408 Aql (=4U 1957+115)
}

\author{
P. A. Mason ${ }^{1,2}$, E. L. Robinson ${ }^{3}$, S. Gomez ${ }^{1}$, J. V. Segura ${ }^{1}$ \\ ${ }^{1}$ Department of Physics, University of Texas at El Paso, 500 W. University, El Paso, TX, USA, 79902 \\ ${ }^{2}$ Department of Mathematics and Physical Sciences, New Mexico State University - DACC, Las Cruces, NM, USA, \\ 88003 \\ ${ }^{3}$ Department of Astronomy, University of Texas at Austin, 1 University Station C1400, Austin, TX, USA, 78712
}

Corresponding author: pmason@nmsu.edu

\begin{abstract}
We present new optical observations of V1408 Aql $(=4 \mathrm{U} 1957+115)$, the only low mass X-ray binary, black hole candidate known to be in a persistently soft state. We combine new broadband optical photometry with previously published data and derive a precise orbital ephemeris. The optical light curves display sinusoidal variations modulated on the orbital period as well as large night to night changes in mean intensity. The amplitude of the variations increases with mean intensity while maintaining sinusoidal shape. Considering the set of constraints placed by the X-ray and optical data we argue that V1408 Aql may harbor a very low mass black hole.

Optical light curves of UW CrB display partial eclipses of the accretion disk by the donor star that vary both in depth and orbital phase. The new eclipses of UW CrB in conjunction with published eclipse timings are well fitted with a linear ephemeris. We derive an upper limit to the rate of change of the orbital period. By including the newly observed type I bursts with published bursts in our analysis, we find that optical bursts are not observed between orbital phases 0.93 and 0.07 , i.e. they are not observable during partial eclipses of the disk.
\end{abstract}

Keywords: low mass X-ray binaries - photometry - individual: V1408 Aql - individual: UW CrB.

\section{Introduction}

Low mass X-ray binaries (LMXBs) are composed of a low mass donor and a compact object, either a black hole or a neutron star primary. The compact star accretes matter from the donor via an accretion disk. We present updates on our previous work on the black hole candidate V1408 Aql and the LMXB containing a neutron star, UW CrB (see Mason et al. 2012).

\section{V1408 Aql}

V1408 Aql is a LMXB whose secondary star fills its Roche Lobe and the compact object is a black hole candidate. This is the only LMXB black-hole candidate known to be in a persistently active soft state. There are only three other X-ray binaries that have been found to be persistently active, LMC X-1, LMC X-3 and Cyg X1. But unlike V1408 Aql, these are all high mass X-ray binaries. V1408 Aql has always remained in the spectrally soft, disk dominated, X-ray state, never reaching the low/hard state. The persistent activity of V1408 Aql and the disk-dominated soft X-ray spectrum are likely to be related.

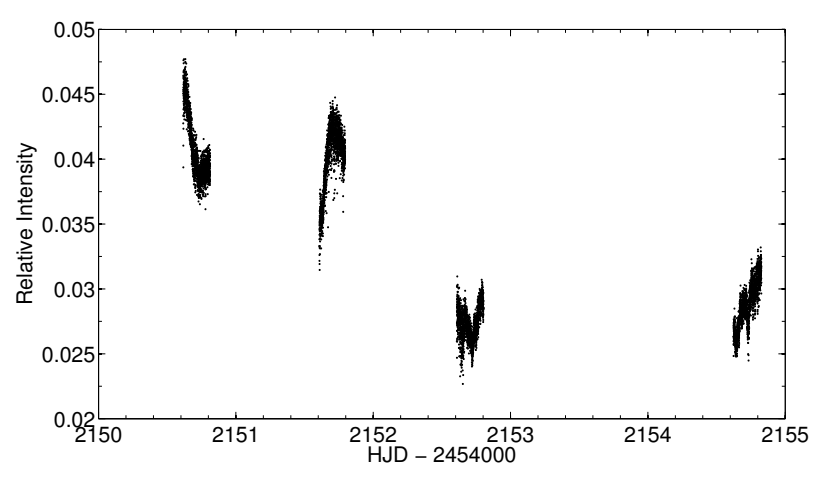

Figure 1: Optical photometry of V1408 Aql on four nights during 10-15 August 2012. Intensity is shown relative to the combined light of stars labeled 6 and 8 of Figure 1 in Bayless et al. (2011). An orbital sine wave like variation is seen each night as well as a large drop in brightness between the second and third night. Once night to night variations are removed, the light curve is nearly sinusoidal.

The optical light curve of V1408 Aql shows a sinusoidal orbital modulation at the system's $9.33 \mathrm{hr}$ orbital 
period. This period was first measured by Thorstensen (1987) and it was later confirmed by Bayless et al. (2011) and Mason et al. (2012).

We obtained new high-speed optical photometry of V1408 Aql using the $2.1 \mathrm{~m}$ Otto Struve telescope of McDonald Observatory on four nights in August 2012 and five nights in July 2012. Photometry was obtained using the Argos CCD photometer (Mukadam and Nather, 2005) and a BVR (broad-band) filter with a time resolution of 10 seconds. The light curves from August 2012 are shown in Figure 1. Strong night to night transitions such as these are often observed. As part of our analysis, nightly mean variations were removed and normalized to form a data set for period analysis. Once normalized and phased the orbital modulation becomes apparent, and a clear sinusoidal modulation is observed.

\subsection{Updated Ephemeris for V1408 Aql}

Using all the available optical photometry for a total of 126 hours of observation spread over 29 nights and 5 years, we construct a phase dispersion minimization periodogram (Stellingwerf, 1978), see Figure 2. Preliminary results were presented by Gomez et al. (2013). We derive a precise orbital period of $0.388893(3)$ days. This improved period is consistent with previously published results. Figure 2 also shows previous period determinations with error bars (Thorstensen 1987; Bayless et al. 2011; Mason et al. 2012). The improved ephemeris for the time of maximum flux is

$$
T(H J D)=2454621.829(4)+0.388893(3) E
$$

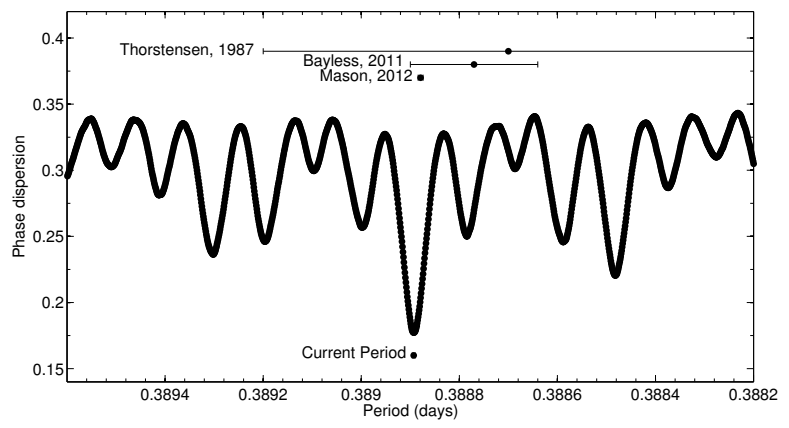

Figure 2: Period search results using phase dispersion minimization. Previously published results are also shown.

\subsection{The Nature of the Compact Star}

Multi-wavelength datasets yield a variety of constraints on the compact component of this binary. The X-ray data show a soft spectrum well described by a multitemperature blackbody and in $15 \%$ of the observations an additional non-thermal steep power law component is seen (Nowak et al. 2012). The optical intensity also occasionally increases sharply; see the histogram of nightly mean brightness in Figure 3. The distance of V1408 Aql is likely $d>7 \mathrm{kpc}$, but the compact star mass remains largely unconstrained by X-ray data (Nowak et al. 2012). Radio emission is 330-810 times fainter than expected in relation to other X-ray binaries (Russell et al. 2011).

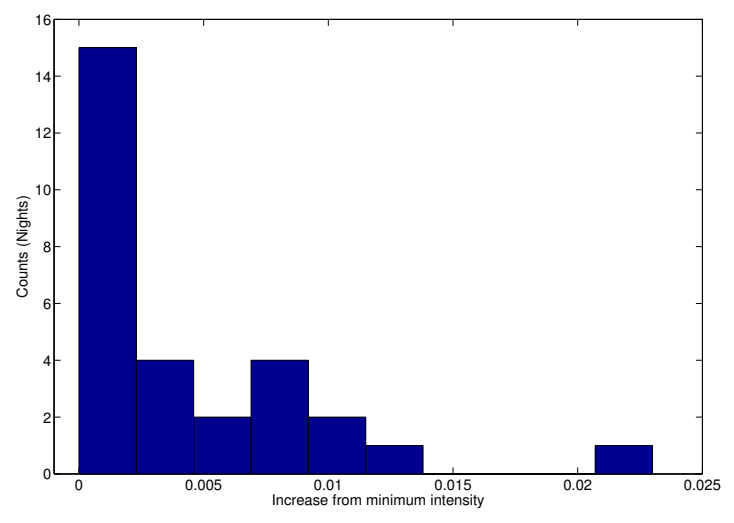

Figure 3: Histogram of nightly optical intensity variations. In order to examine the sinusoidal modulation, light curves were shifted by the amounts shown in the histogram. Zero on this linear scale is the relative intensity of the minimum observed nightly mean. For example, the first night shown in Figure 1 is shifted down by approximately 0.013 to remove the night to night variation. On most nights V1408 Aql remained near minimum intensity, while on one night (14 July 2012) it showed a particularly high intensity.

The optical data suggest that the orbital modulation is dominated by the heated face of the donor star. Even as strong night to night intensity fluctuations occur, a strong sinusoidal modulation remains at all brightness levels. Bayless et al. (2011) estimated a mass ratio of $q=m_{2} / m_{1}>0.3$.

The primary is most likely a black hole, since the system has never shown a type-I X-ray burst since its discovery in 1973, and the black hole candidate has a fast spin and a spectrum typical for black holes (Nowak et al. 2008). Fits of models to the optical light curve give strong evidence for a low mass primary with a mass of $M<7 M_{\odot}$, as well as a low inclination of $i=11^{\circ}-25^{\circ}$. In summary, a low mass black hole model is consistent with both optical and X-ray emission studies of V1408 Aql.

\section{UW CrB}

The LMXB UW CrB contains a neutron star primary. The optical light curve displays partial eclipses of the disk by the donor and frequent bursts (Mason et al. 
2008, 2012, Hakala et al. 2009). The appearance of type I X-ray bursts, either as X-rays or reprocessed as optical emission clearly indicates that the compact object is a neutron star. The secondary star is likely significantly evolved. An HST/COS UV spectrum UW $\mathrm{CrB}$ was obtained (Froning et al. 2012) in which the $\mathrm{C}$ IV lines are totally absent.

We obtained optical photometry at the McDonald Observatory Otto Struve 2.1-m telescope in June 2013, using the Argos CCD photometer (Mukadam and Nather, 2005), a BVR filter, and 10-second time resolution (see Figure 4). Several bursts are easily noticed. The burst frequency varies from night to night.

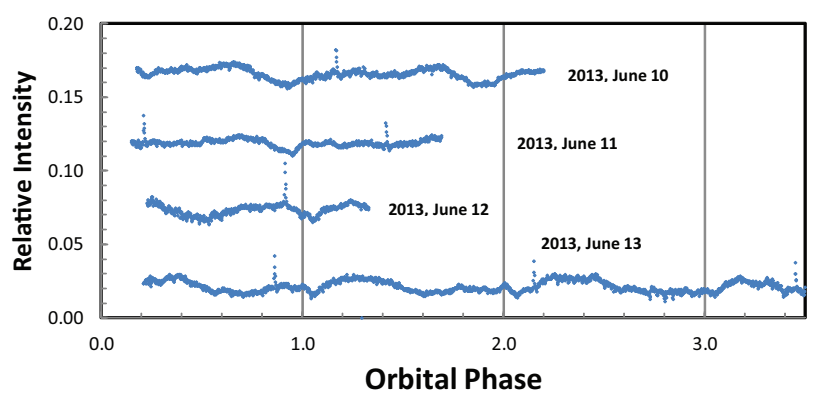

Figure 4: Optical photometry of UW $\mathrm{CrB}$ on four successive nights in June 2013 is shown, compared to star C in the finder chart of Hakala et al. (1998). The light curves taken on the first three nights have been shifted in intensity relative to the fourth by increments of 0.05. The phase and shape of the partial eclipses fluctuate over a 5.5 day beat cycle. See Mason et al. (2008, 2012) and Hakala et al. (2009).

\subsection{An updated ephemeris for UW $\mathrm{CrB}$}

We measured 7 new eclipse times which we combined with 56 eclipses listed by Mason et al. (2008, 2012) for a total of 63 eclipses.

The new best-fit linear ephemeris is:

$$
T(H J D)=24553118.83822(47)+0.077067211(17) E
$$

where $\mathrm{E}$ is the eclipse number. We also fit a quadratic function to the eclipses in the O-C diagram (see Figure 5 ). The quadratic term is not significant, but allows the derivation of an upper limit to the period derivative of (at $90 \%$ confidence):

$$
|d P / d t|<3.0 \times 10^{-11} \mathrm{~s} / \mathrm{s} .
$$

\section{$3.2 \quad$ Optical bursts}

As a mixture of hydrogen and helium accumulates on the neutron star, some hydrogen fuses steadily while a
He-rich layer builds up. When this atmosphere becomes dense and hot enough, it suddenly fuses into carbon and emits a type I X-ray burst (e.g. Strohmayer and Bildsten, 2006).

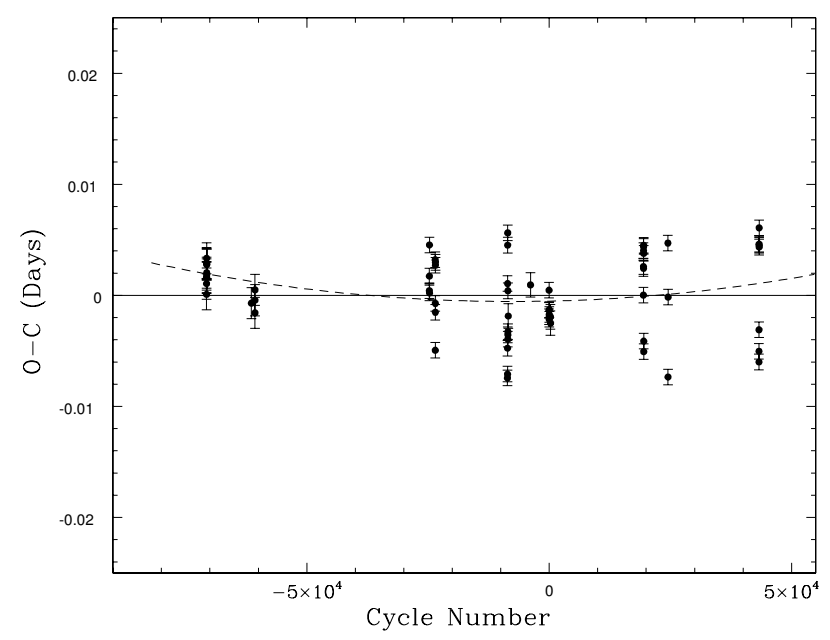

Figure 5: O-C diagram for 63 eclipses of UW $\mathrm{CrB}$ with respect to the best-fit linear ephemeris. The dashed line is the best-fit quadratic function. However, the quadratic term is not statistically significant. The vertical scatter of the points is due to periodic phase shifting of the eclipses.

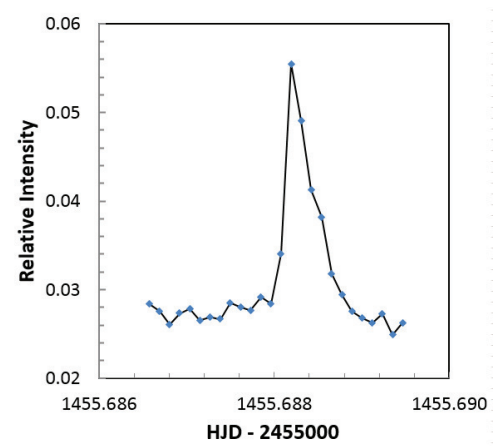

Figure 6: Photometry of a burst that occurred on June 12, 2013. The intensity, compared to star $\mathrm{C}$ in Figure 1 of Hakala et al. (1998), of this typical burst rises to peak in 10-20 s and declines in about $50 \mathrm{~s}$.

UW $\mathrm{CrB}$ is a frequent X-ray burster (Hakala et al., 2005). Optical bursts are observed (e.g. Hynes, Robinson, and Jeffery, 2004) because regions in the accretion disk absorb X-rays and are heated. The heated areas emit optical radiation and we see a burst while the optical emission regions cool. An example optical burst is shown in Figure 6. When combined with published timings (see Mason et al. 2012 and Hakala et al. 2009 and references therein) 40 bursts events have been ob- 
served. These are shown as a function of orbital phase in Figure 7. Notice that there is a partial eclipse zone where no bursts have been detected. If the bursts were being reprocessed off the secondary star, the number and strength of the bursts would have decreased slowly before and increased slowly after eclipses. Instead, the number drops abruptly at the edge of the eclipse. This is strong evidence that the optical burst emission region is the disk and not the heated face of the secondary.

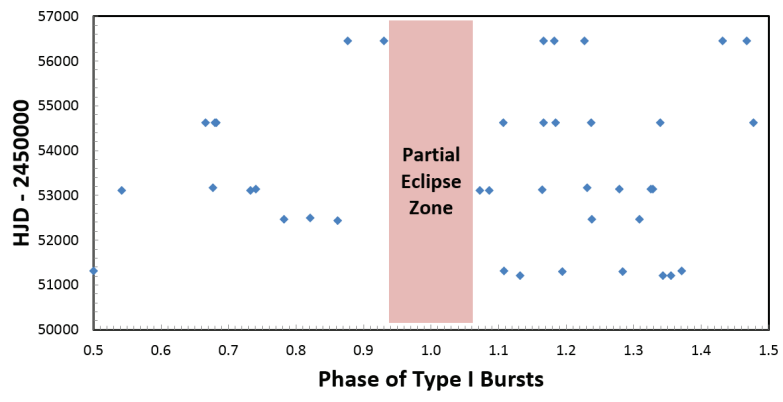

Figure 7: Distribution of 40 type I bursts as a function of phase. No bursts occurred between phase 0.93 and 1.07 due to partial eclipses of the accretion disk by the companion.

\section{Conclusions}

A new ephemeris is derived for V1408 Aql. The light curves show sinusoidal modulation with night-to-night changes in mean brightness. Our models favor a black hole primary with a mass less than 7 solar masses. We suggest that the orbital modulation is caused primarily by the changing aspect of the heated face of the donor star. V1408 Aql (=4U 1957+115) is likely a black hole, and there is increasing evidence that it is near the lower end of the observed black hole mass distribution.

Analysis of new and previously published partial eclipses of the accretion disk in $\mathrm{UW} \mathrm{CrB}$ are well fitted with a linear ephemeris. In addition, there is $~$ 5 day periodic shifting around phase 0 due to precession of an elliptical accretion disk (Mason et al. 2008, 2012; Hakala et al. 2012). An upper limit on the rate of change of the orbital period based on the best fit quadratic ephemeris is derived, see equation 1. Strong bursts are seen at all phases outside of eclipse indicating that the disk is the source of the re-processing responsible for the optical bursts, rather than the heated face of the donor star.

\section{Acknowledgement}

We thank Tom Maccarone for discussions concerning the nature of the compact object in V1408 Aql $(=4 \mathrm{U}$
1957+115). We thank the referee, Hans Ritter, for a careful review and many helpful comments and corrections. This work is supported by NSF/PAARE grant No. 0958783.

\section{References}

[1] Bayless, A. J., Robinson, E. L., Mason, P. A., Robertson, P., 2011, ApJ, 730, 43. doi:10.1088/0004-637X/730/1/43

[2] Froning, C. S., 2012, AAS, 219, 153.12.

[3] Gomez, S., Mason, P. A., Robinson, E. L., 2013, AAS, 221, 142.25.

[4] Hakala, P., et al., 2005, MNRAS, 356, 1133.

[5] Hakala, P., Hjalmarsdotter, L., Hannikainen, D. C., Muhli, P., 2009, MNRAS, 394, 892.

[6] Hynes, R. I., Robinson, E. L., Jeffery, E., 2004, ApJ, 608, 101. doi:10.1086/422471

[7] Mason, P. A., Robinson, E. L., Gray, C. L., Hynes, R. I., 2008, ApJ, 685, 428. doi:10.1086/590381

[8] Mason, P. A., Robinson, E. L., Bayless, A. J., Hakala, P. J., 2012, AJ, 144, 108.

[9] Mukadam, A. S., Nather, R. E., 2005, JApA, 26, 321.

[10] Nowak, M. A., et al., 2008, ApJ, 689, 1199. doi:10.1086/592227

[11] Nowak, M. A., Wilms, J., Pottschmidt, K., Schulz, N., Maitra, D., Miller, J., 2012, ApJ, 744, 107. doi:10 . 1088/0004-637X/744/2/107

[12] Russell, D. M., et al., 2011, ApJ, 739, L19. doi:10.1088/2041-8205/739/1/L19

[13] Stellingwerf, R. F., 1978, ApJ, 224, 953.

[14] Strohmayer, T., Bildsten, L., 2006, in "Compact Stellar X-Ray Sources", ed. by W. Lewin and M. Van der Klis, (Cambridge: Cambridge Univ. Press) p. 113. doi:10.1017/CB09780511536281.004

[15] Thorstensen, J. R., 1987, ApJ, 312, 739.

\section{DISCUSSION}

SIMONE SCARINGI: Could the absence of type I $\mathrm{X}$-ray bursts at specific phases be used to constrain the size of the secondary star and/or the emitting region giving rise to the burst. 
P. A. Mason et al.

PAUL MASON: Yes, especially when combined with exclude significant re-processing off of the companion information about the mass ratio. Currently, we do con- star.

strain the optical burst emission region to the disk. We 